\title{
Analysis of Handover Planning in Wideband Code Division Multiple Access (W-CDMA)
}

\author{
${ }^{1}$ Win Adiyansyah Indra, ${ }^{1}$ Saravanan $\mathrm{A} / \mathrm{L}$ Sukumaran, ${ }^{2} \mathrm{~K}$ halil Azha Mohd Annuar and ${ }^{2}$ Irianto \\ ${ }^{1}$ Department of Electronic and Computer Engineering Technology, Melaka, Malaysia \\ ${ }^{2}$ Department of Electrical Engineering Technology, Faculty of Engineering Technology, \\ Universiti Teknikal Malaysia Melaka, Hang Tuah Jaya, 76100 Durian Tunggal, Melaka, Malaysia
}

\begin{abstract}
This study analysed the most suitable handover type for Wideband Code Division Multiple Access (W-CDMA) system by using simulation software. Forsk Atoll, Earth Explorer and Global Mapper 15 were used to do the simulations, local area (BukitBeruang, $2.240^{\circ} \mathrm{N} 102.279^{\circ} \mathrm{E}$ to Bukit Baru, $2.222^{\circ} \mathrm{N} 102.275^{\circ} \mathrm{E}$ ) was selected based on the its varies altitudes, high density, high traffic, etc. The simulation result showed that by implementing soft handover in W-CDMA system, the users will experience no drop call during handover from one cell to another cell. The Received Signal Strength Indicator (RSSI) range value between -80 to $-100 \mathrm{dBm}$ in all selected area which considered as good signal strength range. There's no blank spot. Physical measurement in the field (selected area), like drive test, particularly in the handover areas, borders of every cells is required to verify the simulation results, the data from physical measurement in the field must be considered and key in inside the simulation software, so that, the revised or new results will have a high validity.
\end{abstract}

Key words: Handover, Wideband Code Division Multiple Access (W-CDMA), Atoll, Received Signal Strength Indicator (RSSI), cells, implementing

\section{INTRODUCTION}

Handover is one of the important aspect or technique to enhance the performance of networks. Handover is the process that transfers an ongoing call from one cell to another when a user moves through the coverage area of a cellular networks. The handover can be classified into two types. The first one is the hard handover. Hard handover is supported by Time Division Multiple Access (TDMA), Frequency Division Multiple Access (FDMA) and Code Division Multiple Access (CDMA) systems (Pasandideh and St-Hilaire, 2013). Hard handover is a break-before make method, means a new channel is set up after the release of the old channel. On the other hand for hard handover a certain amount of margin may be introduced to remove the Ping-Pong effect. In a W-CDMA cellular network, the soft handover is essential which used make-before-break method. This is due to W-CDMA users in the same cell share the same frequency spectrum simultaneously. For instance, if the pilot signal from a new Base Station is stronger than the threshold value of T_ADD (Adding Threshold), it establish a new link to the base station while keeping the existing link. For this situation, the call is said to be in soft handover (Guo et al., 2003).
There are different types of challenges in the planning of handover in Wideband Code Division Multiple Access (W-CDMA) technology. From network provider's point of view, it would be simpler for them to investigate those issues by using a specially designed troubleshooting tool or with improvements to the existing tools. This research will analyses and propose better planning in handover for W-CDMA technology which is strongly driven by the required network capacity and quality of service. These considerations in turn influence network parameters such as transmit power and antenna height. In W-CDMA system, it shares the same frequency band between all cells (Ouyang and Fallah, 2009). This means the bandwidth is utilised efficiently. Therefore, the frequency reuse happens to differentiate the signal which consists of the similar frequency. The Handover situation considered when a mobile change its frequency. The main concern of a handover is to make sure that there is always has network connection with the old Base Station (BS) as the new Base Station (BS) that has ability to control the network connection without any connection loss. This method always maintaining a network connection without any disturbance with one or more Base Station (BS) simultaneously (Prabhakar, 2013). So that, the mobile user can continue their telephone conversation even though they are travelling.

Corresponding Author: Win Adiyansyah Indra, Department of Electronic and Computer Engineering Technology, Melaka, Malaysia 
W-CDMA handover types: Handover plays a vital role for W-CDMA and any type of cellular telecommunications system. Most of the subscribers always not aware of the changes happen in W-CDMA Handover. Whenever happen failures within the W-CDMA handover will cause to drop calls that will resulted in user dissatisfaction and it may cause the subscriber to use different networks, so that it produces higher churn rate. There are number of different types of handover but in this section, I would like discuss about the three important W-CDMA handover, such as hard handover, soft handover and softer handover. The chances for causing more reliable forms of handover became possible which resulted in one of a choice of various types of handover are available depending upon the different situations. Air interface on CDMA2000 1x system provides the ability to handover for voice service and data services (St-Hilaire et al., 2006).

Hard handover: The hard handover defined as there is a "hard" change during the process of handover. For hard handover, the radio connections are always broken and need to re-connect again. Even though hard handover is consistent but some time it also produce a short break in the connection. The basic consideration of a hard handover is relatively straightforward. The hard handover can be happen by following some basic stages such as:

- The network determines the handover by considering strength of signal and strength of broadcast channels

- If the connection between node B and the UE is broken

- If a new connection formed between node B and the User Equipment (UE)

The major problem is that problem in re-establishing the connection will cause the handover to fail and the call or connection to drop (Schinnenburg et al., 2003). A handover commonly used if the quality of the connection of the power of the received pilot between the node B and the UE is keep dropping and it is possibly produce handover to another cell with good radio characteristics. The $2 \mathrm{G}$ systems is similar as GSM, the handover process disturbs the connection for a short period of time, not identified by the end user of an existing connection and it changes with a new network connection to a new cell where the user is handed over with a different frequency. This process known as hard handover. The cell where the user is handed over is called as a target cell. Based on theoretical, all cells in W-CDMA use the similar frequency range, therefore, in $3 \mathrm{G}$ systems it is possibly to have the connection to the new cell before changing to the current cell and make sure at least one radio link with a node B. This concept is called as soft handover. Hard Handover is used in $3 \mathrm{G}$ systems when it is needed to change the carrier frequency, when performing inter-frequency handover. The W-CDMA carrier frequency change for balancing load or when performing Inter-RAT (Radio Access Technology). W-CDMA hard handovers can be used in a several circumstances:

- Condition when one cell moving to an adjacent cell on different frequency

- Implementation of a mode change from like FDD to TDD mode

- Condition when cell moving from one cell to another where the channel is without any capacity and the frequency need to change

The problems facing W-CDMA hard handovers similar as GSM (Ismail and Aripin, 2005). If the usage level are high, the capacity of a selected cell that a User Equipment (UE) want to enter may be not enough to support a new subscriber. To avoid this, it need to be reserve some capacity for new subscribers. It can be accomplished by spreading the loading if necessary. For instance UEs that can accept a required high signal from a neighbouring cell may be transmitted as the original cell nears its capacity level.

Soft handover: Soft handover is a type of handover that was produced by the evolution of CDMA. Soft handover happens when a UE is in the coverage area of two cells. It connects to the two base stations which can be worked at the same time and it also, ensures that UE can communicate with two nearby base stations. For connection up to one link during the handover process, it will produces a more dependable and easy way to perform handover. Besides, the soft handover can uses more simultaneous links. So that, the adjacent cells need to be working on the same frequency or channel as UEs do not have more than one transmitters and receivers that is needed if they were using different frequency values (Lee and Kang, 2000).

When the UE and node B undertake a soft handover, the UE can obtains signals from the two node $B$ and merges them using the RAKE receiver capability available in the signal processing of the UE. For the uplink the condition is become more difficult if the signal merging cannot be assigned in the node $\mathrm{B}$ as more than one node $\mathrm{B}$ is involved. Instead, merging is complete on a frame by frame basis. The good frames are selected after each interleaving period. The selection is completed by considering the outer loop power control algorithm which 


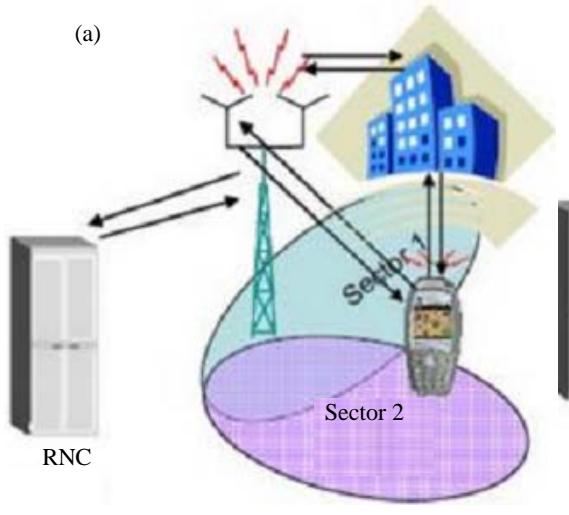

Fig. 1a, b): Comparison between softer and soft handover

determines the Signal to Noise Ratio (SNR) of the received uplink signals. This information is probably used to choose the best quality frame. If the soft handover process has been accomplished, the connection links to the old node $\mathrm{B}$ are changed and the UE continues to communicate with the new node $\mathrm{B}$.

The soft handover consumes a higher degree of the network resources than a common link and also hard handover. However, this can be enhance by the improved performance and reliability of the handover process. But with around $5-10 \%$ of handovers dropping into this range, network operators must be to consider for it. A rake receiver is a type of radio receiver that has been made feasible in connection link by the use the concept of Digital Signal Processing (DSP) (Wong and Lim, 1997). It is usually used to avoid the problems of multipath propagation. Then, it accomplishes this by using some kind sub-receivers known as "fingers" which are given a certain multipath component. Furthermore, each sub-receiver then processes its component and decodes it. The outputs from the sub-receiver are then mixed together to produce the maximum contribution from each path. Therefore, the rake receivers and multipath propagation can be used to generate signal to noise (Schinnenburg et al., 2003).

Softer handover: It is a type of handover known as softer handover. The softer handover happens if the new radio links are combined from the similar node B. This happens when some sectors may be served from the same node B which simplifying the combining as it can be accomplished within the node $\mathrm{B}$ and not require connection further back into the network. W-CDMA softer handover is only use whenever a UE can generate the signals from two sectors served by the same node B. This may happen as a result of the sectors overlapping or more generally resulted of multipath propagation resulting

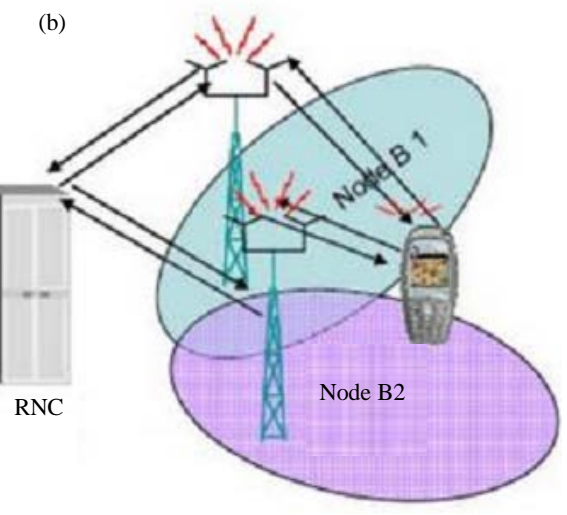

from reflections from buildings. For the uplink, the signals received by the node $B$, the signals from the two sectors can be routed to the similar RAKE receiver and then merged to produce an improved signal. The downlink, it is a little more difficult because the various sectors of the node $B$ use various scrambling codes. To avoid this, different sub-receiver of the RAKE receiver apply the selected de-spreading or de-scrambling codes to the received signals. If this has been done, than only they can be merged as before.

In a nutshell in W-CDMA systems there are two types of handover such as soft and softer handover. These type of handovers can perform at least two simultaneous connections with different node B like Soft handover or by using the same node $\mathrm{B}$ when multi-path propagation between the UE and node $\mathrm{B}$ causes the node $\mathrm{B}$ to obtain the signal sent from the UE from of different segment. Besides, the UE for soft and softer handover always keeps at least one radio link connection to the (Universal Terrestrial Radio Access Network) also, known as UTRAN. Figure 1 illustrates the concept of soft and softer handover.

The active set is plays a vital role in order to save the track of the number link connection. The active set is also called Ericsson handover is the cells sets used in a certain UE connection. Besides, the UEs radio link produced to cells present in Active sets. The set will update systematically known as event based whenever a connection is active, depend on ability of the Primary Common Pilot Channel (P-CPICH) Ec/Io or the Primary Common Pilot Channel (P-CPICH) RSCP (Received Signal Code Power). Related to Ericsson handover is known as the cell which is the one of the active set have a $\mathrm{P}-\mathrm{CPICH}$ with the good quality $\mathrm{Ec} / \mathrm{No}$. For a node $\mathrm{B}$, the requested an incoming handover same as an incoming call, even though the RRM algorithms can change whether the request receives from a Handover Connection 
or not, as it is the condition in the Ericsson RRM algorithms. For instance given that using handover in a correct way leads to a development in capacity because of the soft hand over gain, the handover connections have low probability to block than new incoming non-handover calls. In addition, this specification can be illustrate $\mathrm{n}$ the Ericsson diagrams where various thresholds for blocking are set-depending if the connection is established for voice or not established for handover or non-handover call. The problem to establish a new connection in the target cell is referred to as a "handover failure" and it happens when no new resources are available in the target cells or when the radio link quality has drops below acceptable levels before the call could be handed-over. The first cause leads to handover blocked attempts and the last one leads to handover dropped attempts and both are good measures of the handover performance in the network. A single transmitter is used in between UE, only one power control loop is active. This may not be optimal for all circumstances but it simplifies the hardware and basic operation (Yang et al., 2002).

\section{MATERIALS AND METHODS}

The planning of handover in W-CDMA system process can be categorized into different phases (Bernaschi et al., 2004). The first phase is the pre-planning phase. In this phase requirement of services and basic network configuration parameters are investigated. Next, the second phase is location-planning phase. In this phase, the research area andpossible location to set up the base station are investigated. The data identified whether related to the geographical properties and the assumed traffic volumes at various point of the area will be incorporated into a digital map which comprises of different size of pixel that keeps all the information about the location point. Based on the digital map, computer simulation will identify the different possibilities to set up the radio network by using some of optimization algorithms. The main goal is to achieve soft handover. The important consideration of handover depends on the traffic and the capacity. The traffic determines the quality of service whereas the capacity determines the population of people in selected location. Then, the third phase is called handover planning. In this phase the simulated results will be analysed and compared with previous analysis until the expected results is achieved. The planning of W-CDMA is a difficult task. In order to make the planning task simpler, the planning problem is usually categorised into three sub problems. The sub problems are the access network planning, the core network planning andthe cell planning. There are two methods to solve these problems which are sequential method and global method. For sequential method, the problems are considered separately whereas more than one problem considered at a time for global method (Salkola, 2002).

There were three softwares used such as Earth Explorer, Global Mapper 15 and Forsk Atoll. The stated softwares are used at different stages. For Digital Map, the software used for this stage is called Earth Explorer. Earth Explorer is used to explore the world's largest collection of images of the Earth's surfaces. The images are in forms such as satellite images, elevation and land cover datasets anddigitized maps. The digital maps of earth explorer in the format of Digital Raster Graphics (DRGs). A Digital Raster Graphic (DRG) is used to scan image standard series topographic map of a USGS by including information all map collar. The image inside the map neat line is Geo referenced to the surface of the earth which fit to projection of the Universal Transverse Mercator (UTM). Since, the Earth Explorer Software is an online software, therefore, we need the internet connection in order to get the map. In order to download digital map, we can use software called Global Mapper 15. The last and main software is Forsk Atoll, Forsk Atoll is a radio planning and optimisation software which can run network cell planning where it imported Geo data map and all the cellular parameters, like antenna height, location, power, distance, etc were simulated.

\section{RESULTS AND DISCUSSION}

The selected area for this W-CDMA handover planning area is surrounding Bukit Beruang $\left(2.240^{\circ} \mathrm{N}\right.$ $\left.102.279^{\circ} \mathrm{E}\right)$ to Bukit Baru $\left(2.222^{\circ} \mathrm{N} 102.275^{\circ} \mathrm{E}\right)$. Bukit Beruang is selected due to its highly dense-populated area in Melaka Multimedia University (MMU) is the famous landmark in Bukit Beruang and most students are living there. On the other hand, Malacca Manipal Medical College is located in Bukit Baru which also, consists of high population. Thus, the demanding for network services is higher in the selected location and require to have continous strong signal. Therefore, these locations are selected as the most suitable location for handover planning. The altitude of Bukit Beruang ranging 33-155 m (Bukit Beruang Hill). Bukit Beruang is known for it's hill which have a rounded elevation of limited extent rising above the surrounding land with local relief of $<160 \mathrm{~m}$. For Bukit Baru, the altitude is about $17.78 \mathrm{~m}$. Due to different altitude, the planning of locating antenna should considered the height of location. For the higher zones, the antenna coverage is wider whereas for the lower land 


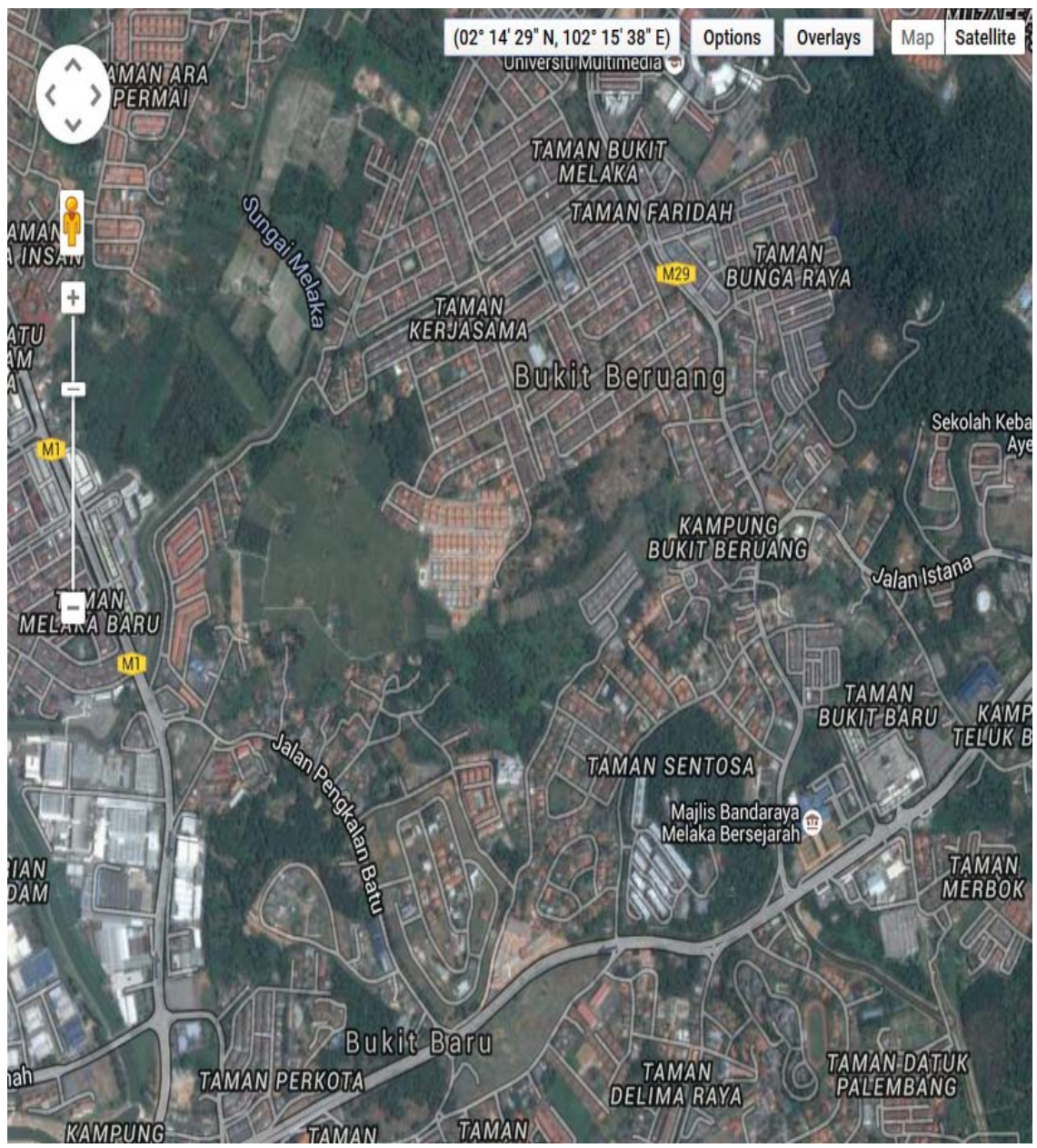

Fig. 2: Satellite map location of Bukit Beruang to Bukit Baru

zones the antenna placing by arranging in hexagonal cells. Three hexagonal cells are sharing one antenna. Each hexagon radius is about $400 \mathrm{~m}$ (Fig. 2).

It is necessary to identify the altitude of the location before placing the antenna. On to that, the number of antenna using also needs consideration which should be sufficient to the selected location. The main concern of this research is on good network quality and capacity by concerning on handover. Since, this research related to $3 \mathrm{G}$ network planning, therefore the soft handover is used which mainly refers to feature of W-CDMA standards. The soft handover is subdivided into several handover status called softer handover, soft handover, softer-softer handover, softer-soft handover, soft-soft handover and blank spot. By depending on the prediction of the active set size of the terminal and service capabilities.

Figure 3 showed the simulation result for handover planning of the selected location at different altitudes. The altitude ranging from more 1000 to $<100 \mathrm{~m}$. The highest point of the altitude located at top of Bukit Beruang Hill which shown by BTS51 in Fig. 2. The highest altitude point is covered by red colour. At the highest altitude point, one BTS is required to cover wider coverage zone. The handover status is classified into six categories such as blank spot, softer handover, soft handover, softer-softer handover, softer-soft handover andsoft-soft handover. Each category of handover is labelled by using different colours. For the altitude, it had been planned to place less antenna on higher altitude and more antenna on lower ground. This is because a higher altitude can cover more areas compared to lower altitude. Besides, it ensures that good network quality and capacity can be provided to the selected location users. Each hexagonal cells consists of the stated handovers. This proves that there are no signal losses between each cells of different network range. Not enough than that, Fig. 4 shows the traffic density map. The traffic handover happens when the quality or the strength of the radio signal falls below certain parameters specified by RNC. 


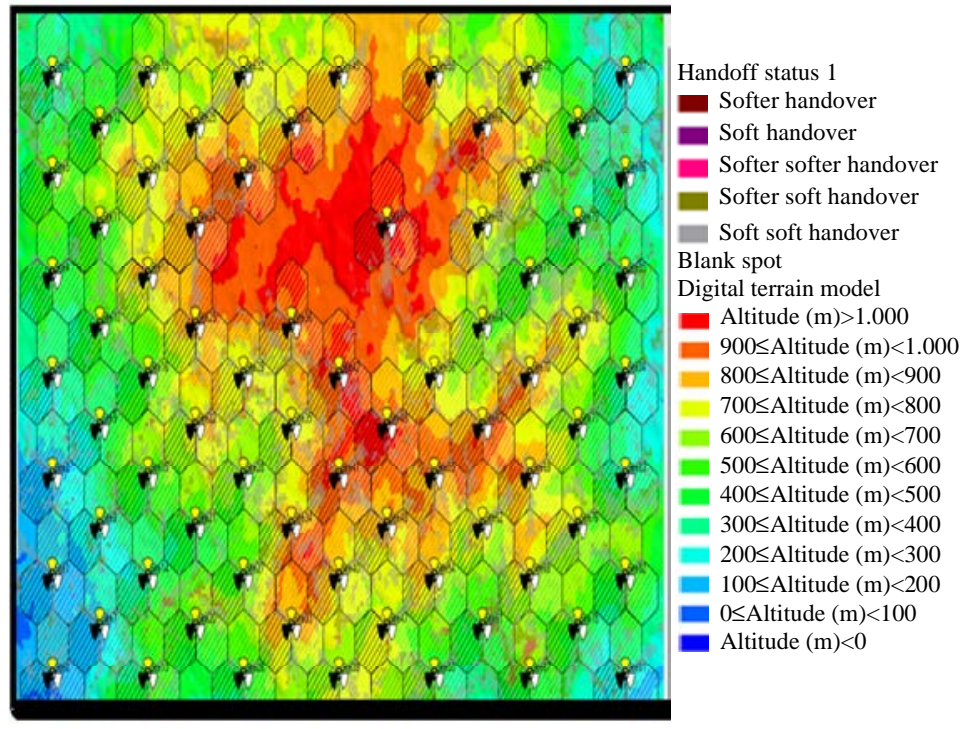

Fig. 3 : Handover planning at different altitudes

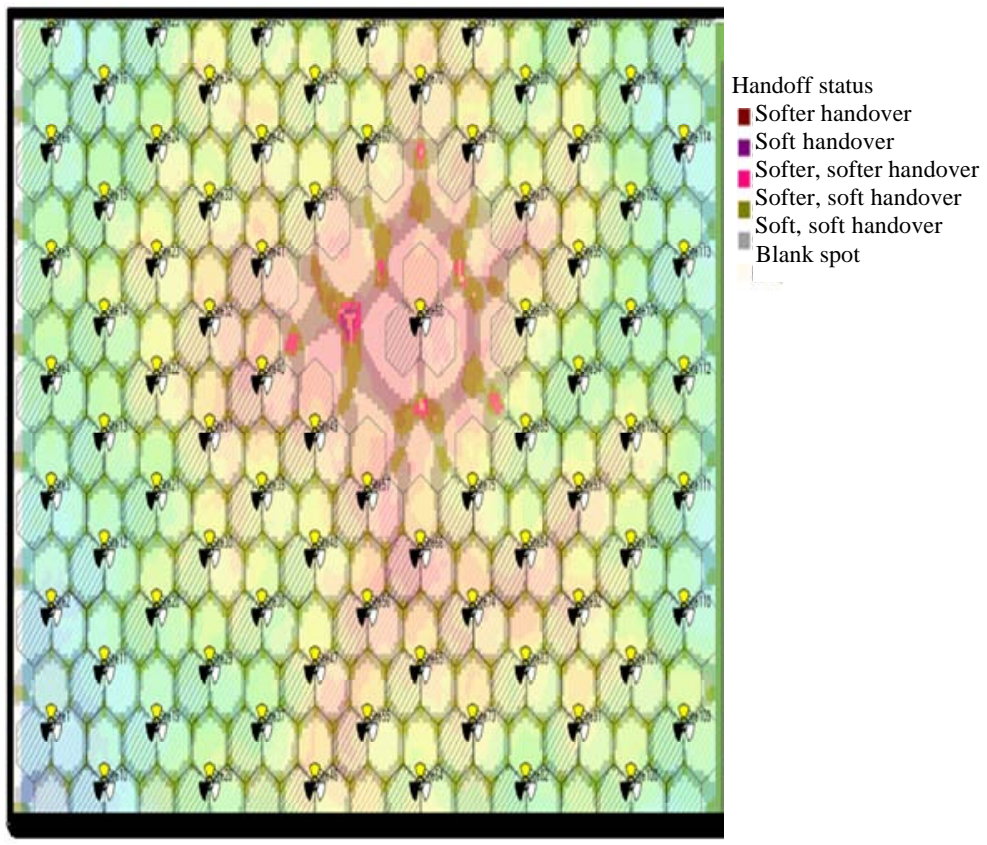

Fig. 4 : Traffic density of handover planning

The atoll simulation of traffic density which used to plan handover status are shown in Fig. 4. Similar with previous figure, the handover status is categorised into 6 types, such as softer handover, soft handover, softer-softer handover, softer-soft handover, soft-soft handover and blank spot. From the obtained results, there are 58 antennas located in between 175 sectorized hexagonal cells. Based on Fig. 4, the occurance of softer-softer handover is higher compared to other handover status. In addition, there were no blank spot zones. For traffic handover, the implementation for planning by placing the antenna exactly on same location as did in altitude handover planning before. Thus, it can be concluded that the traffic handover also, produces same signal strength as altitude handover without any signal loss.

Figure 5 showed cell phone reception signal or also, known as signal strength received by mobile phone from nearest base station. Based on result obtained the signal strength value range from -80 to $-120 \mathrm{dBm}$. Most of 


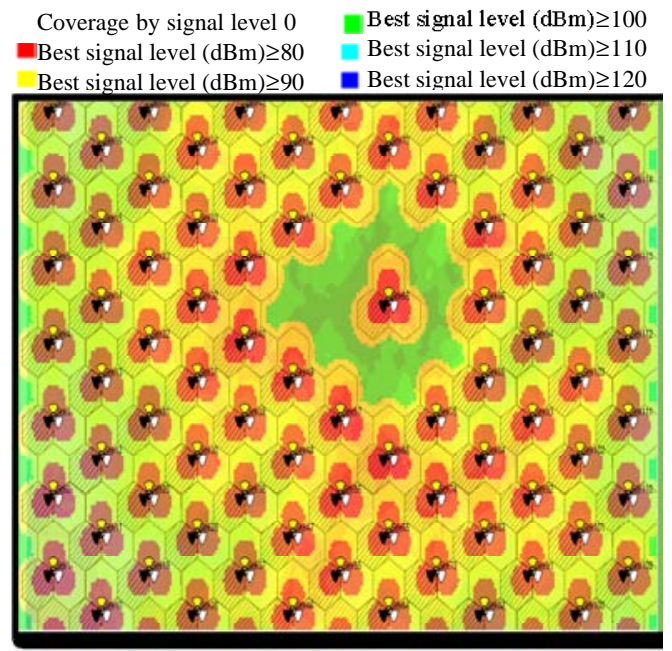

Fig. 5: Signal strength $(\mathrm{dBm})$ at selected location signals strength are $<-80 \mathrm{dBm}$. This implies that, good signal strength exists at the selected location. Thus, less chance for signal loss and provide continous signal to the users.

Figure 6 illustrated the location where the Base Stations are needed to be placed in order to achieve the desired output result. The satellite map above shows exactly same location where decided for handover planning. The satellite map consists of 58 base stations located in between 175 sectorized hexagonal cells. Furthermore, Fig. 5 also illustrated the accurate location of BTSs which are free from obstacles, like buildings. So that, it can avoid shadowing (log normal fading). Most of BTSs are located at the forest sites and empty land. This helps to prevent Radio Frequency (RF) radiation.

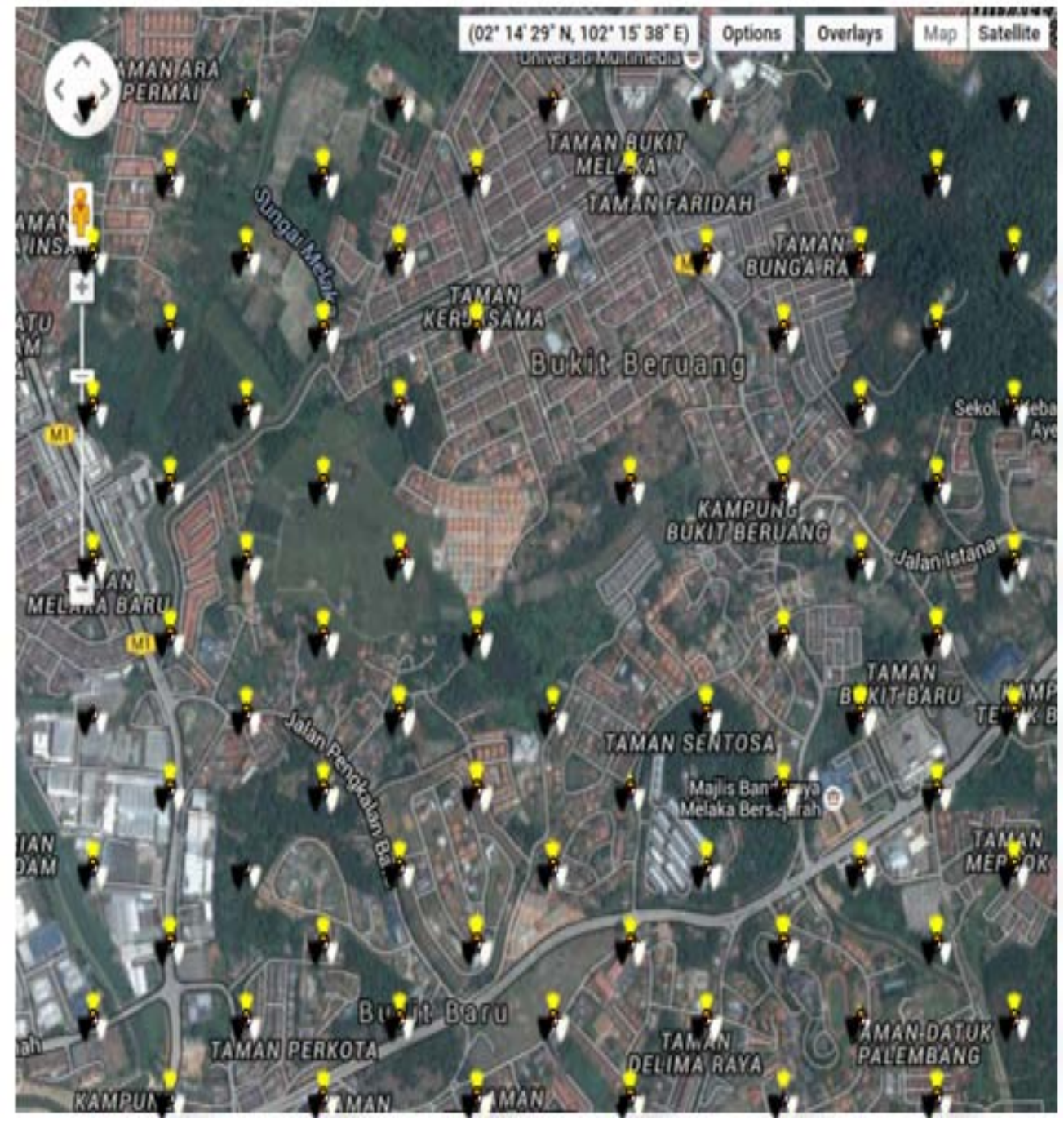

Fig. 6: Antennas placement at selected location 


\section{CONCLUSION}

This study proposed soft handover as the most suitable handover types for W-CDMA cellular system. Implementation of handover planning significantly can produce good quality of signal with better signal strength. The value of signal strength ranging from -80 to $100 \mathrm{dBm}$ which considered as good signal strength. Soft handover refers to a feature used by the W-CDMA standards, so that, a cell phone is simultaneously connected to two or more cells during a call.

\section{LIMITATIONS}

Limitations were experienced during the project, such as the limited features of Forsk Atoll Software, limited function on calculating handover status, Forsk Atoll Software only supports certain map formats to import. There are less choices of softwares made for handover planning. Therefore, it really requires a complete software to calculate the handover status to find the accurate output results for a better planning.

\section{RECOMMENDATIONS}

Future research is verification or validation of the simulation results that come up with soft handover is the best handover type for W-CDMA against the real application in the fields, measurement in the fields, particularly during handover at the border of every cells. And also, the use of sophisticated handover softwares would give a precise, accurate results.

\section{ACKNOWLEDGEMENTS}

The researchers would like to thank for the support given to this research by Ministry of Higher Education Malaysia and Universiti Teknikal Malaysia Melaka (UTeM) for supporting this under GrantRAGS/1/2015/ TK0/FTK/03/B00118 project.

\section{REFERENCES}

Bernaschi, M., F. Cacace and G. Iannello, 2004. Vertical handoff performance in heterogeneous networks. Proceedings of the Workshops on Mobile and Wireless Networking/High Performance Scientific, Engineering Computing/Network Design and Architecture/Optical Networks Control and Management/Ad Hoc and Sensor Networks/Compil, August 18, 2004, IEEE, Montreal, Canada, ISBN: 07695-219 8-3, pp: 100-107.
Guo, L., J. Zhang and C. Maple, 2003. Coverage and capacity calculations for $3 \mathrm{G}$ mobile network planning. Comput. Inf. Syst., 2000: 16-17.

Ismail, M. and N. Aripin, 2005. Downlink soft handover performance for different cell selection schemes in WCDMA system. Proceedings of the 200513 th and 7th Joint IEEE/Malaysia International Conference on Networks and Communic, November 16-18, 2005 , IEEE, Kuala Lumpur, Malaysia, ISBN: 1-4244-0000-7, pp: 808-811.

Lee, C.Y. and H.G. Kang, 2000. Cell planning with capacity expansion in mobile communications: A Tabu search approach. IEEE. Trans. Veh. Technol., 49: 1678-1691.

Ouyang, Y. and M.H. Fallah, 2009. Evolving core networks from GSM to UMTS R4 version. Intl. J. Mobile Network Des. Innovation, 3: 93-102.

Pasandideh, M.R. and M. St-Hilaire, 2013. Automatic planning of 3G UMTS all-IP release 4 networks with realistic traffic. Comput. Oper. Res., 40: 1991-2003.

Prabhakar, L., 2013. A comparative study of three TDMA digital cellular mobile systems (GSM, IS-136 NATDMA and PDC) based on radio aspect. Editorial Preface, 4: 139-143.

Salkola, M.I., 2002. CDMA capacity-can you supersize that?. Proceedings of the 2002 IEEE Conference on Wireless Communications and Networking Conference Record(WCNC 2002; Cat. No.02TH8609), March 17-21, 2002, IEEE, Orlando, Florida, USA., ISBN:0-7803-7376-6, pp: 768-773.

Schinnenburg, M., I. Forkel and B. Haverkamp, 2003. Realization and optimization of soft and softer handover in UMTS networks. Proceedings of the 2003 5th Conference on European Personal Mobile Communications (Conf. Publ. No. 492), April 22-25, 2003, IET, Glasgow, UK., ISBN:0-85296-753-5, pp: $22-25$.

St-Hilaire, M., S. Chamberland and S. Pierre, 2006. Uplink UMTS network design-an integrated approach. Comput. Networks, 50: 2747-2761.

Wong, D. and T.J. Lim, 1997. Soft handoffs in CDMA mobile systems. IEEE. Pers. Commun., 4: 6-17.

Yang, X., S. Ghaheri-Niri and R. Tafazolli 2002. UTRA soft handover optimisation. Proceedings of the 3rd International Conference on $3 \mathrm{G}$ Mobile Communication Technologies, May 8-10, 2002, IET, London, UK, ISBN:0-85296-749-7, pp: 37-41. 